\title{
PHENOMENOLOGICAL THEORY OF MULTIMODE SURFACE WAVES FOR PLANE STRUCTURES* $\dagger$
}

\author{
BY \\ SAMUEL N. KARP AND FRANK C. KARAL, JR. \\ Courant Institute of Mathematical Sciences, New York University
}

\begin{abstract}
The notion of impedance boundary condition is generalized and illustrated by the discussion of the electromagnetic field arising from a magnetic line dipole source located above a plane structure. The generalized impedance boundary condition contains a set of parameters whose totality offers a physical description of the configuration. By suitably selecting these parameters, the configuration may be made to correspond to a structure that supports surface waves. We give an exact solution for a plane structure that supports one, two and three surface waves. The magnitudes of the surface waves are obtained and simple formulas for the radiated far field patterns are given. We also show how the methods employed can be extended to the case of any number of surface waves. This involves an $n$th order mixed boundary condition for a second order partial differential equation.
\end{abstract}

1. Introduction. It is well known that surface waves can be excited and propagated along particular idealized electromagnetic structures. Examples are dielectric coated surfaces, dielectric slabs and corrugated or laminated structures.** In so far as the surface wave feature is concerned, it is known that the details of the structure may be suppressed and replaced by an impedance boundary condition [9]. Subsequent developments showed that the use of impedance boundary conditions facilitated the solution of more complex problems such as the excitation, propagation and diffraction of surface waves on structures with discontinuities in impedance and geometry [5, 6, 15-24]. The phenomenological representation by an impedance boundary condition is also useful in analyzing the effect of other structures such as absorbers and surfaces with anisotropic conductivity $[6,16]$.

Since an open structure acts to some extent like a wave guide, and since its theory is of use in surface wave antennas, it would seem to be of interest to generalize the existing theory to multi-mode surface wave guides. This means to generalize the body of theory mentioned above to the case of surfaces that support more than one surface wave. Thus one should ultimately possess a body of theory for surface wave guides analogous to that already available for conventional wave guides. Typical problems of interest are the analysis of the effects of radiation from junctions, changes in cross section, effects of terminations, etc. The present paper is the first part of a series devoted to these topics.

*Received August 16, 1965; revised manuscript received January 21, 1966. The material discussed in this paper is a condensed version of Report No. EM-198 referred to in our paper "Phenomenological Theory of Multimode Surface Wave Structures", Proceedings of the Symposium on Quasi-Optics, Vol. XIV, pp. 465-472. (New York: John Wiley and Sons, Inc., 1964).

$\dagger$ This research was supported by the Air Force Cambridge Research Laboratories, Office of Aerospace Research, under Contract No. AF 19(628)3868. Reproduction in whole or in part is permitted for any purpose of the U. S. Government.

**The Bibliography given at the end of the paper is representative but not complete. Additional references are given by Collin [7], Cottony [8] and Zucker [15]. 
The phenomenological theory on which the generalization is based has not been verified experimentally. However, it is clear that such surfaces can be realized, at least by sufficiently thick dielectric slabs or coatings. There are also certain qualitative features of the results for the phenomenological theory of one mode in the case of the excitation problem which are in agreement with the exact theoretical results for a slab or thin coating. It would certainly be worthwhile to compare the exact theory with the phenomenological theory presented here with more than one mode and in detail. Also, experimental results for one or more modes would also be desirable. The phenomenological theory offers the possibility of analytical solutions of complex problems whose analysis from the standpoint of a given detailed structure is difficult or impossible by present analytical methods. Another problem is the correspondence between detailed properties of the structure and the choice of a generalized boundary condition. The problem has been treated for corrugated structures which support one surface wave. [7]. It is also well understood in the case of a dielectric structure for one mode and has been partially analyzed by the authors (in a paper to appear shortly [26]) for a multi-mode dielectric structure. In brief, it would seem desirable to compare the phenomenological theory (i.e., exact solutions using generalized boundary conditions) with exact solutions in which the detailed structure is taken into account more explicitly.

The purpose of the present paper is to study the electromagnetic field that arises when a magnetic line dipole source is located above a plane structure that supports several surface waves if the surface of the structure is characterized by a generalized impedance boundary condition. This condition is introduced here for the first time. We describe a procedure for finding the exact solution for plane structures that support any number of surface waves. We then give the exact solutions for the cases of one, two and three surface waves. A different procedure, which is elementary in character, is next described to find the radiated far field and radiation pattern for any number of surface waves. Results are then given for the cases of one, two and three surface waves. It should be emphasized that the effects of terminations, finiteness, etc., which constitute the main applications of the present formulation, are excluded from consideration in this introductory paper. The treatment of these basic diffraction effects, along the lines of $[5,6,15-24]$, are the subject of subsequent papers.

The boundary condition we employ to describe the propagation of several surface waves along a plane surface wave structure is given by

$$
\prod_{i=1}^{n}\left(\frac{\partial}{\partial y}+\lambda_{i}\right) u(x, y)=0, \quad y=0
$$

where $u(x, y)$ is the $z$-component of the magnetic vector $\mathbf{H}(x, y), x$ and $y$ are the usual Cartesian rectangular coordinates, and $\lambda_{i}$ are constants characteristic of the surface. The value of $\lambda_{i}$ is given by

$$
\lambda_{i}=i \omega \epsilon Z_{i}=i \omega \epsilon\left(R_{i}-i X_{i}\right),
$$

where $\epsilon$ is the permittivity of free space, $\omega$ is the angular frequency, and $Z_{i}, R_{i}$ and $X_{i}$ are the impedances, resistances and reactances of the surface, respectively. For surface wave propagation it is necessary to require that*

$$
\operatorname{Re} \lambda_{i}>0 \text {. }
$$

*This assumption is employed in the illustrative examples that follow, but is not an essential restriction. 
It is worth noting that if there is to be negligible attenuation of the surface waves along the surface, it is necessary to require that $R_{i} \ll X_{i}$. This boundary condition is a natural generalization of the usual impedance boundary condition employed on surfaces supporting one surface wave. For example, when $n=1$ in (1.1) we obtain the boundary condition

$$
\left(\frac{\partial}{\partial y}+\lambda_{1}\right) u(x, y)=0, \quad y=0 .
$$

This boundary condition is well known and is a good approximation to several important physical configurations. Perhaps the simplest example is a thin dielectric layer lying on a perfectly conducting ground plane. This and other possible physical configurations are discussed in [7], where additional references are given. It is clear that a surface wave solution of the form

$$
u_{s}(x, y)=A_{1} e^{-\lambda_{1} y+i \sqrt{k^{2}+\lambda_{1}^{2}}|x|}
$$

satisfies the wave equation, is outgoing at infinity, and satisfies (1.4). Hence a surface characterized by (1.4) can indeed support surface waves. Similarly, the generalized boundary condition (1.1) can also support surface waves of the form

$$
u_{s}(x, y)=\sum_{i=1}^{n} A_{i} e^{-\lambda_{i} y+i \sqrt{k^{2}+\lambda_{i}^{2}|x|}} .
$$

Therefore (1.1) is the natural extension to structures supporting several surface waves. We shall call these multi-mode surface wave structures and use the generalized boundary condition (1.1) as their defining property. We will not discuss the physical realizability of the surface wave structures here. We point out, however, that a perfectly conducting ground plane with a sufficiently thick dielectric coating, or several different thin dielectric coatings, will support more than one surface wave.

2. Multi-mode surface wave structures. We examine the electromagnetic field that arises when a magnetic line dipole source is located above a plane structure that supports $n$ surface waves. Consider the plane surface $y=0$ and suppose a magnetic line dipole is located at the point $x=0, y=y_{0}$. See Figure 1 . Assume the region $y \geq 0$ is free space. The boundary condition on the surface is given by the impedance condition

$$
\prod_{i=1}^{n}\left(\frac{\partial}{\partial y}+\lambda_{i}\right) u(x, y)=0, \quad y=0
$$

where $u(x, y)$ is the magnetic field component $H_{z}$ and the $\lambda_{j}$ are constants characteristic of the surface material. We wish to solve the time-reduced Maxwell's equations, subject to the prescribed condition, and obtain the amplitude of the surface waves propagated along the surface.

The time dependent form of Maxwell's equations is

$$
\left.\begin{array}{l}
\nabla \times \mathbf{H}=-i \omega \mathrm{E} \\
\nabla \times \mathbf{E}=i \omega \mu \mathbf{H}
\end{array}\right\}
$$

where $\mathbf{E}$ and $\mathbf{H}$ are the electric and magnetic field intensities, and $\epsilon$ and $\mu$ are the permittivity and magnetic permeability of free space. We assume the time dependence to be of the form $e^{-i \omega t}$. Because of the geometry, the field produced is independent of $z$ and 


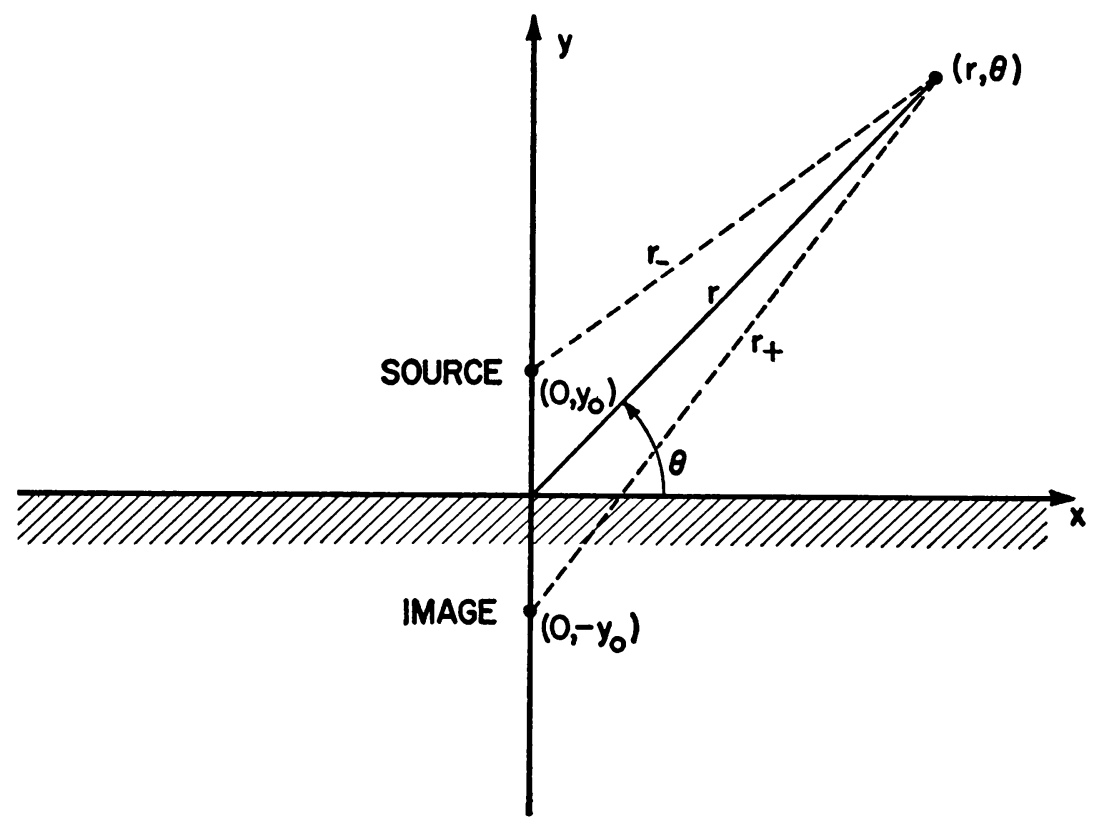

Fig. 1. Magnetic line dipole source and image in the presence of a surface characterized by a generalized impedance boundary condition.

hence is completely determined by the value of $H_{z} \equiv u(x, y)$. We have

$$
H_{x}=H_{y}=E_{z}=0
$$

and

$$
\left.\begin{array}{l}
E_{x}=-\frac{1}{i \omega \epsilon} \frac{\partial u}{\partial y} \\
E_{\nu}=+\frac{1}{i \omega \epsilon} \frac{\partial u}{\partial x}
\end{array}\right\} .
$$

The function $u \equiv u(x, y)$ satisfies the wave equation

$$
\left(\nabla^{2}+k^{2}\right) u=-4 \pi \delta(x-0) \delta\left(y-y_{0}\right)
$$

where $\nabla^{2}$ is the rectangular Laplacian, $k$ is the propagation constant of free space and $\delta$ is the Dirac delta function. Therefore, the mathematical problem reduces to that of solving the inhomogeneous wave equation (2.5) subject to the mixed boundary condition given by (2.1). In addition to the prescribed boundary condition, we require the far field to be outgoing, and the value of the total magnetic field (excluding the source) to be finite everywhere.

We now employ a technique developed by the authors [5, 6, 16-22] for use in solving mixed boundary value problems arising in electromagnetic diffraction theory. Introduce an auxiliary function $v$ defined by

$$
v=\prod_{i=1}^{n}\left(\frac{\partial}{\partial y}+\lambda_{i}\right) u
$$


Then $v$ satisfies the equation

$$
\left(\nabla^{2}+k^{2}\right) v=-4 \pi \prod_{j=1}^{n}\left(-\frac{\partial}{\partial y_{0}}+\lambda_{j}\right) \delta(x-0) \delta\left(y-y_{0}\right)
$$

where we use the result

$$
\frac{\partial}{\partial y} \delta\left(y-y_{0}\right)=-\frac{\partial}{\partial y_{0}} \delta\left(y-y_{0}\right) .
$$

Also, $v$ is now subject to the simpler conditions

(1) $\quad v=0, \quad y=0$

(2) Outgoing traveling waves at $\infty\}$.

The solution of the auxiliary problem is easily constructed by introducing the image located at the point $x=0, y=y_{0}$. The desired solution is

$$
v(x, y)=i \pi \prod_{i=1}^{n}\left(-\frac{\partial}{\partial y_{0}}+\lambda_{i}\right)\left\{H_{0}^{(1)}\left(k r_{-}\right)-H_{0}^{(1)}\left(k r_{+}\right)\right\} .
$$

where

$$
r_{ \pm}=\sqrt{x^{2}+\left(y \pm y_{0}\right)^{2}} .
$$

Here $r_{-}$and $r_{+}$are the distances from the source and image to the observation point, respectively. Clearly the solution vanishes on the boundary, is outgoing at infinity, and possesses the correct singular behavior at the source $x=0, y=y_{0}$. A particular solution of the original field $u$ can be found by solving (2.6). It is

$$
u_{p}(x, y)=\sum_{i=1}^{n} \prod_{\substack{i=1 \\(i \neq j)}}^{n} \frac{1}{\left(\lambda_{i}-\lambda_{i}\right)} e^{-\lambda_{i} y} \int_{-\infty}^{y} e^{\lambda_{i} \eta} v(x, \eta) d \eta .
$$

This solution is not a wave function since the $x$-derivative in regions including the positive $y$-axis is discontinuous. We can remedy this defect, however, by adding to (2.11) complementary solutions of the form

$$
u_{c}(x, y)=\sum_{i=1}^{n} A_{i} e^{-\lambda_{i} y+i \sqrt{k^{3}+\lambda_{i}^{3}|x|}} .
$$

Then the combined solution given by

$$
u(x, y)=u_{p}(x, y)+u_{c}(x, y)
$$

can be made to satisfy the continuity condition on $\partial u / \partial x$. This is equivalent to imposing the jump condition

$$
\left[\frac{\partial u}{\partial x}\right]=\operatorname{Lim}_{x \rightarrow 0}\left\{\frac{\partial u}{\partial x}(x>0, y)-\frac{\partial u}{\partial x}(x<0, y)\right\}=0 .
$$

The requirement (2.14) is sufficient to determine the constants $A_{i}$, which are the amplitudes of the surface waves. The exact solution for $u(x, y)$ will contain the sum or difference of the source and image (depending on whether or not there are an odd or even number of surface wave modes, respectively), a linear combination of integrals, all of the same type but with different $\lambda_{i}$ 's, and a sum of surface waves as given by (2.12). 
3. Summary of Results for $n=1,2,3$. Using the procedure indicated in the preceding Section, we list the results for 1, 2 and 3 surface wave modes. In obtaining the final expressions for $u(x, y)$, we employ integration by parts and use the following results:

$$
\begin{gathered}
\frac{\partial}{\partial x} H_{0}^{(1)}(k r)=-k H_{1}^{(1)}(k r) \cos \theta \\
\operatorname{Lim}_{x \rightarrow 0} \int_{-\infty}^{\nu} e^{\lambda n} H_{1}^{(1)}\left(k \sqrt{x^{2}+\left(\eta+y_{0}\right)^{2}}\right) \frac{x d \eta}{\sqrt{x^{2}+\left(\eta+y_{0}\right)^{2}}}=-\frac{2 i}{k} e^{-\lambda y_{0}}
\end{gathered}
$$

Also, we find it convenient to introduce the integral

$$
I\left(\lambda_{i} ; x, y\right)=\lambda_{i} e^{-\lambda_{i} y} \int_{-\infty}^{y} e^{\lambda_{i} \eta} H_{0}^{(1)}\left(k \sqrt{x^{2}+\left(\eta+y_{0}\right)^{2}}\right) d \eta .
$$

The results for $u(x, y)$ are summarized below:

One Mode $(n=1)$

$$
\begin{aligned}
u(x, y)=i \pi\left\{H_{0}^{(1)}\left(k r_{-}\right)+H_{0}^{(1)}\left(k r_{+}\right)\right\}-2 \pi i I\left(\lambda_{1} ; x, y\right) & \\
& +\frac{4 \pi i \lambda_{1}}{\sqrt{k^{2}+\lambda_{1}^{2}}} e^{-\lambda_{1}\left(y+y_{0}\right)+i \sqrt{k^{2}+\lambda_{1}{ }^{2}|x|}}
\end{aligned}
$$

Two Modes $(n=2)$

$$
\begin{aligned}
u(x, y) & =i \pi\left\{H_{0}^{(1)}\left(k r_{-}\right)-H_{0}^{(1)}\left(k r_{+}\right)\right\}-2 \pi i \frac{\left(\lambda_{1}+\lambda_{2}\right)}{\left(\lambda_{2}-\lambda_{1}\right)} I\left(\lambda_{1} ; x, y\right) \\
& -2 \pi i \frac{\left(\lambda_{1}+\lambda_{2}\right)}{\left(\lambda_{1}-\lambda_{2}\right)} I\left(\lambda_{2} ; x, y\right)+\frac{4 \pi i \lambda_{1}\left(\lambda_{1}+\lambda_{2}\right)}{\sqrt{k^{2}+\lambda_{1}^{2}}\left(\lambda_{2}-\lambda_{1}\right)} e^{-\lambda_{1}\left(y+y_{0}\right)+i \sqrt{k^{2}+\lambda_{1}}|x|} \\
& +\frac{4 \pi i \lambda_{2}\left(\lambda_{1}+\lambda_{2}\right)}{\sqrt{k^{2}+\lambda_{2}^{2}}\left(\lambda_{1}-\lambda_{2}\right)} e^{-\lambda_{2}\left(y+y_{0}\right)+i \sqrt{k^{2}+\lambda_{2}^{2}}|x|}
\end{aligned}
$$

Three Modes $(n=3)$

$$
\begin{aligned}
u(x, y) & =i \pi\left\{H_{0}^{(1)}\left(k r_{-}\right)+H_{0}^{(1)}\left(k r_{+}\right)\right\}-2 \pi i \frac{\left(\lambda_{1}+\lambda_{2}\right)\left(\lambda_{1}+\lambda_{3}\right)}{\left(\lambda_{2}-\lambda_{1}\right)\left(\lambda_{3}-\lambda_{1}\right)} I\left(\lambda_{1} ; x, y\right) \\
& -2 \pi i \frac{\left(\lambda_{1}+\lambda_{2}\right)\left(\lambda_{2}+\lambda_{3}\right)}{\left(\lambda_{1}-\lambda_{2}\right)\left(\lambda_{3}-\lambda_{2}\right)} I\left(\lambda_{2} ; x, y\right)-2 \pi i \frac{\left(\lambda_{1}+\lambda_{3}\right)\left(\lambda_{2}+\lambda_{3}\right)}{\left(\lambda_{1}-\lambda_{3}\right)\left(\lambda_{2}-\lambda_{3}\right)} I\left(\lambda_{3} ; x, y\right) \\
+ & \frac{4 \pi i \lambda_{1}\left(\lambda_{1}+\lambda_{2}\right)\left(\lambda_{1}+\lambda_{3}\right)}{\sqrt{k^{2}+\lambda_{1}^{2}}\left(\lambda_{2}-\lambda_{1}\right)\left(\lambda_{3}-\lambda_{1}\right)} e^{-\lambda_{1}\left(y+y_{0}\right)+i \sqrt{k^{2}+\lambda_{1}{ }^{2}}|x|} \\
+ & \frac{4 \pi i \lambda_{2}\left(\lambda_{1}+\lambda_{2}\right)\left(\lambda_{2}+\lambda_{3}\right)}{\sqrt{k^{2}+\lambda_{2}^{2}}\left(\lambda_{1}-\lambda_{2}\right)\left(\lambda_{3}-\lambda_{2}\right)} e^{-\lambda_{2}\left(y+y_{0}\right)+i \sqrt{k^{2}+\lambda_{2}{ }^{2}}|x|} \\
+ & \frac{4 \pi i \lambda_{3}\left(\lambda_{1}+\lambda_{3}\right)\left(\lambda_{2}+\lambda_{3}\right)}{\sqrt{k^{2}+\lambda_{3}^{2}}\left(\lambda_{1}-\lambda_{3}\right)\left(\lambda_{2}-\lambda_{3}\right)} e^{-\lambda_{3}\left(y+y_{0}\right)+i \sqrt{k^{2}+\lambda_{2}{ }^{2}}|x|}
\end{aligned}
$$

4. Determination of the radiated far field. We now determine the radiated far field arising from a magnetic line dipole source located above a plane surface that sup- 
ports $n$ surface waves. The radiated far field can be found in the usual way by expanding the exact solution asymptotically for large distances. We shall not proceed in this manner, however, but will use a more direct and elementary procedure $[5,6,16-22]$ that does not require the exact results obtained in the earlier Sections.

Consider a plane structure that supports $n$ surface waves. The same physical configuration and notation employed earlier applies here also. See Figure 1. Rewriting (2.6) and (2.9) we have

$$
v=\prod_{i=1}^{n}\left(\frac{\partial}{\partial y}+\lambda_{i}\right) u
$$

and

$$
v=i \pi \prod_{i=1}^{n}\left(-\frac{\partial}{\partial y_{0}}+\lambda_{i}\right)\left\{H_{0}^{(1)}\left(k r_{-}\right)-H_{0}^{(1)}\left(k r_{+}\right)\right\}
$$

Let $r=\sqrt{x^{2}+y^{2}}$ be the distance measured from the origin. Then the far field expressions for $r_{ \pm}$become

$$
r_{ \pm} \approx r \pm y_{0} \sin \theta
$$

provided $y_{0} \ll r$. When $k r_{ \pm}$is large, (4.2) becomes

$$
v(r, \theta) \approx \sqrt{\frac{8 \pi}{k r}} e^{i(k r-\pi / 4)} \prod_{i=1}^{n}\left(-\frac{\partial}{\partial y_{0}}+\lambda_{i}\right) \sin \left(k y_{0} \sin \theta\right)
$$

where we use the asymptotic expansion of the Hankel function for large argument. We expect the radiated far field of $u(r, \theta)$ to have a similar form, except that it will contain surface wave terms that are appreciable at large $x$ near the surface $y=0$. Hence, for fixed $\theta$ and large $r$, we assume

$$
u(r, \theta) \approx \frac{f(\theta)}{\sqrt{r}} e^{i k r}, \quad \theta \neq 0, \quad \theta \neq \pi
$$

where $f(\theta)$ is unknown. Substituting (4.4) and (4.5) into (4.1), and using the result

$$
\frac{\partial}{\partial y}=\sin \theta \frac{\partial}{\partial r}+\frac{1}{r} \cos \theta \frac{\partial}{\partial \theta} \approx \sin \frac{\partial}{\partial r},
$$

we obtain $f(\theta)$ correct to terms of order $r^{-1 / 2}$. The expression for the radiated far field then becomes

$$
u(r, \theta) \approx \sqrt{\frac{8 \pi}{k r}} e^{i(k r-\pi / 4)} \frac{\prod_{j=1}^{n}\left(-\frac{\partial}{\partial y_{0}}+\lambda_{i}\right) \sin \left(k y_{0} \sin \theta\right)}{\prod_{j=1}^{n}\left(i k \sin \theta+\lambda_{j}\right)}
$$

The radiation pattern of the far field is easily determined from (4.7). We define the pattern function to be

$$
F\left(\theta, \frac{\lambda_{1}}{k}, \frac{\lambda_{2}}{k}, \cdots, \frac{\lambda_{n}}{k}, k y_{0}\right) \equiv\left|\sqrt{\frac{k r}{8 \pi}} e^{-i(k r-\pi / 4)} u(r, \theta)\right|^{2}
$$


Then

$$
F\left(\theta, \frac{\lambda_{1}}{k}, \frac{\lambda_{2}}{k}, \cdots, \frac{\lambda_{n}}{k}, k y_{0}\right)=\frac{\left[\prod_{i=1}^{n}\left(-\frac{1}{k} \frac{\partial}{\partial y_{0}}+\frac{\lambda_{i}}{k}\right) \sin \left(k y_{0} \sin \theta\right)\right]^{2}}{\prod_{i=1}^{n}\left[\left(\frac{\lambda_{i}}{k}\right)^{2}+\sin ^{2} \theta\right]}
$$

The expressions for the radiated far fields and the pattern functions for the cases $n=1,2$ and 3 are summarized below:

One Mode $(n=1)$

$$
\begin{gathered}
u(r, \theta) \approx \sqrt{\frac{8 \pi}{k r}} e^{i(k r-\pi / 4)} \frac{\left[\lambda_{1} \sin \left(k y_{0} \sin \theta\right)-k \sin \theta \cos \left(k y_{0} \sin \theta\right]\right.}{\left[i k \sin \theta+\lambda_{1}\right]} \\
F\left(\theta, \frac{\lambda_{1}}{k}, k y_{0}\right)=\frac{\left[\frac{\lambda_{1}}{k} \sin \left(k y_{0} \sin \theta\right)-\sin \theta \cos \left(k y_{0} \sin \theta\right)\right]^{2}}{\left[\left(\frac{\lambda_{1}}{k}\right)^{2}+\sin ^{2} \theta\right]}
\end{gathered}
$$

Two Modes $(n=2)$

$$
\begin{aligned}
u(r, \theta) \approx & \sqrt{\frac{8 \pi}{k r}} e^{i(k r-\pi / 4)} \\
& \frac{\left[\left(\lambda_{1} \lambda_{2}-k^{2} \sin ^{2} \theta\right) \sin \left(k y_{0} \sin \theta\right)-\left(\lambda_{1}+\lambda_{2}\right) k \sin \theta \cos \left(k y_{0} \sin \theta\right)\right]}{\left[i k \sin \theta+\lambda_{1}\right]\left[i k \sin \theta+\lambda_{2}\right]}
\end{aligned}
$$

$F\left(\theta, \frac{\lambda_{1}}{k}, \frac{\lambda_{2}}{k}, k y_{0}\right)$

$$
=\frac{\left[\left(\frac{\lambda_{1} \lambda_{2}}{k^{2}}-\sin ^{2} \theta\right) \sin \left(k y_{0} \sin \theta\right)-\left(\frac{\lambda_{1}}{k}+\frac{\lambda_{2}}{k}\right) \sin \theta \cos \left(k y_{0} \sin \theta\right)\right]^{2}}{\left[\left(\frac{\lambda_{1}}{k}\right)+\sin ^{2} \theta\right]\left[\left(\frac{\lambda_{2}}{k}\right)^{2}+\sin ^{2} \theta\right]}
$$

Three Modes $(n=3)$

$$
\begin{gathered}
u(r, \theta) \approx \sqrt{\frac{8 \pi}{k r}} e^{i(k r-\pi / 4)}\left[i k \sin \theta+\lambda_{1}\right]^{-1}\left[i k \sin \theta+\lambda_{2}\right]^{-1}\left[i k \sin \theta+\lambda_{3}\right]^{-1} \\
\cdot\left[\left\{\lambda_{1} \lambda_{2} \lambda_{3}-\left(\lambda_{1}+\lambda_{2}+\lambda_{3}\right) k^{2} \sin ^{2} \theta\right\} \sin \left(k y_{0} \sin \theta\right)\right. \\
\left.-k \sin \theta\left\{\left(\lambda_{1} \lambda_{2}+\lambda_{1} \lambda_{3}+\lambda_{2} \lambda_{3}\right)-k^{2} \sin ^{2} \theta\right\} \cos \left(k y_{0} \sin \theta\right)\right] \\
F\left(\theta, \frac{\lambda_{1}}{k}, \frac{\lambda_{2}}{k}, \frac{\lambda_{3}}{k}, k y_{0}\right)=\left[\left(\frac{\lambda_{1}}{k}\right)^{2}+\sin ^{2} \theta\right]^{-1}\left[\left(\frac{\lambda_{2}}{k}\right)^{2}+\sin ^{2} \theta\right]^{-1}\left[\left(\frac{\lambda_{3}}{k}\right)^{2}+\sin ^{2} \theta\right]^{-1} \\
\cdot\left[\left\{\frac{\lambda_{1} \lambda_{2} \lambda_{3}}{k^{3}}-\left(\frac{\lambda_{1}}{k}+\frac{\lambda_{2}}{k}+\frac{\lambda_{3}}{k}\right) \sin ^{2} \theta\right\} \sin \left(k y_{0} \sin \theta\right)\right. \\
\left.-\left\{\left(\frac{\lambda_{1} \lambda_{2}}{k^{2}}+\frac{\lambda_{1} \lambda_{3}}{k^{2}}+\frac{\lambda_{2} \lambda_{3}}{k^{2}}\right)-\sin ^{2} \theta\right\} \sin \theta \cos \left(k y_{0} \sin \theta\right)\right]^{2}
\end{gathered}
$$

\section{BibLiography}

[1] Barlow, H. M., and Cullen, A. L., Surface Waves, Proc. Inst. Elec. Engrgs. Part III, Vol. 100, 1953 , p. 329.

[2] Barlow, H. M., and Karbowiak, A. E., An Experimental Investigation of the Properties of Corrugated Cylindrical Surface Waveguides, Proc. Inst. Elec. Engrgs. Part III, Vol. 101, 1954, pp. 182-188. 
[3] Bazer, J., and Karp, S. N., Propagation of Plane Electromagnetic Waves Past a Shoreline, Jour. of Res. Nat. Bureau of Standards, Vol. 66D, 1962, pp. 319-334.

[4] Brick, D. B., The Radiation of a Hertzian Dipole over a Coated Conductor, Proc. Inst. Elec. Engrgs., Vol. 102C, 1954, pp. 392-295.

[5] Chu, T. S., Kouyounjain, R. G., Karal, F. C. and Karp, S. N., The Diffraction of Surface Waves by a Terminated Structure in the Form of a Right-Angle Bend, I.R.E. Trans. on Antennas and Propagation, Vol. AP-10, 1962, pp. 679-686.

[6] Chu, T. S. and Karp, S. N., The Field of a Dipole Above an Infinite Corrugated Plane, Quart. of Appl. Math. Vol. 21, 1964, pp. 257-268.

[7] Collin, R. E., Field Theory of Guided Waves, McGraw-Hill Book Company, New York, 1960, Chapter 11.

[8] Cottony, H. V. et. al., Antennas and Waveguides and Annotated Bibliography, I.R.E. Trans. on Antennas and Propagation, Vol. AP-7, 1959, pp. 87-98.

[9] Cullen, A. L., The Excitation of Plane Surface Waves, Proc. Inst. Elec. Engrgs. Part IV, Vol. 101, 1954, pp. 225-234.

[10] Felsen, L. B., Diffraction by an Imperfectly Conducting Wedge, McGill Symposium on Microwave Optics, Part II, 1959, pp. 287-292.

[11] Felsen, L. B., Field Solutions for a Class of Corrugated Wedges and Cone Surfaces, Polytech. Inst. Brooklyn, Memo No. 32, July, 1957.

[12] Fernando, W. M. G., and Barlow, H. M., An Investigation of the Properties of Radical Cylindrical Surface Waves Launched over Flat Reactive Surfaces, Proc. Inst. Elec. Engrgs., Vol. 103B, 1956, pp. 307-318.

[13] Friedman, B., and Williams, W. E., Excitation of Surface Waves, Proc. Inst. Elec. Engrgs., Vol. 105C, 1958, pp. 252-258.

[14] Grünberg, G. A., Suggestions for a Theory of Coastal Refraction, Physical Rev., Vol. 63, 1943, pp. $185-189$.

[15] Kane, J., A Surface Wave Antenna as a Boundary Value Problem, Electromagnetic Theory and Antennas, Edited by E. C. Jordan, Pergamon Press, New York, 1963, pp. 891-894.

[16] Karal, F. C., and Karp, S. N., Diffraction of a Skew Plane Electromagnetic Wave by an absorbing Right Angles Wedge, Comm. Pure Appl. Math., Vol. ii, 1958, pp. 495-533.

[17] Karal, F. C., and Karp, S. N., Diffraction of a Plane Wave by a Right Angled Wedge which Sustains Surface Waves on One Face, Quart. Appl. Math., Vol. 20, 1962, pp. 97-102.

[18] Karal, F. C., Karp, S. N., Chu, T. S. and Kouyoumjian, R. G., Scattering of a Surface Wave by a Discontinuity in the Surface Reactance on a Right Angled Wedge, Comm. Pure Appl. Math., Vol. 14, 1961, pp. 35-48.

[19] Karp, S. N., Two Dimensional Green's Function for a Right Angled Wedge Under an Impedance Boundary Condition, New York Univ., Inst. Math. Sci., Div. Electromagnetic Res., Res. Rep. EM-129, 1959.

[20] Karp, S. N., and Karal, F. C., Vertex Excited Surface Waves on Both Faces of a Right Angled Wedge, Comm. Pure Appl. Math., Vol. 12, 1959, pp. 435-455.

[21] Karp, S. N., and Karal, F. C., Vertex Excited Surface Waves on One Face of a Right Angled Wedge, Quart. Appl. Math., Vol. 18, 1960, pp. 235-243.

[22] Karp, S. N., and Karal, F. C., A New Method for the Determination of Far Fields with Application to the Problem of Radiation of a Line Source at the Tip of an Absorbing Wedge, I.R.E. Trans. on Antennas and Propagation, Toronto Symposium on Electromagnetic Theory, Vol. AP-7, 1959, pp. S91-S102.

[23] Kay, A. F., Scattering of a Surface Wave by a Discontinuity in Reactance, I.R.E. Trans. on Antennas and Propagation, Vol. AP-7, 1959, pp. 22-31.

[24] Maluzhinets, G. D., The Excitation, Reflection and Emission of Surface Waves from a Wedge with Given Face Impedances, Soviet Physics, Dokl, Vol. 3, 1958, pp. 752-755.

[25] Zucker, F. J., Progress During the Three Years in Surface and Leaky Wave Antennas, Jour. Res. Nat. Bureau of Standards, Vol. 64D, 1960, pp. 746-750.

[26] Karp, S. N., and Karal, F. C., Generalized Impedance Boundary Conditions with Applications to Surface Wave Structures, to appear in the Proceedings of the Delft Symposium on Electromagnetic Theory (U.R.S.I. Comm. VI Conference, Delft, the Netherlands, 1965). 